\title{
Comparison of the impact of radiotherapy and radiochemotherapy on the quality of life of I-year survivors with cervical cancer
}

This article was published in the following Dove Press journal:

Cancer Management and Research

5 July 2011

Number of times this article has been viewed

\author{
Marianthi Krikeli \\ Maria T Ekonomopoulou ${ }^{2}$ \\ loannis Tzitzikas ${ }^{3}$ \\ Antonios Goutzioulis ${ }^{4}$ \\ Kyriaki Mystakidou ${ }^{5}$ \\ Kyriaki Pistevou-Gombaki ${ }^{3}$ \\ 'Department of Radiation Oncology, \\ Theagenio Cancer Hospital, \\ Thessaloniki, Greece; ${ }^{2}$ Laboratory \\ of General Biology and Genetics, \\ Medical School, Aristotle University \\ of Thessaloniki, Thessaloniki, \\ Greece; ${ }^{3}$ Radiation-Oncology \\ Department, AHEPA University \\ Hospital, Thessaloniki, Greece; \\ ${ }^{4} 4$ th Department of Obstetrics and \\ Gynecology, Aristotle University of \\ Thessaloniki, Thessaloniki, Greece; \\ ${ }^{5}$ Pain Relief and Palliative Care \\ Department, Areteion Hospital, \\ School of Medicine, University of \\ Athens, Athens, Greece
}

Correspondence: Maria T Ekonomopoulou Laboratory of General Biology and Genetics, Medical School, Aristotle

University of Thessaloniki, 54I24

Thessaloniki, Greece

$\mathrm{Tel}+302310999016$

Fax +302310999019

Email biomary@med.auth.gr

\begin{abstract}
Improvement of screening programs and new treatment strategies against cervical cancer (CC) have increased survival rates of patients in the last decades. As more women survive this type of cancer, their quality of life (QOL) has become a field of great scientific and social importance. Different types of therapy have varying results on the QOL of patients. In this study, we compared the impact of radiotherapy (RAD) and radiochemotherapy (RAD/ CHEM) on CC patients' QOL. Our sample included 105 women who suffered from CC stages IA-IIIA. They were treated either with RAD or RAD/CHEM, and filled in the questionnaires 1 year after treatment completion. We used 4 questionnaires, EORTC QLQ C-30, EORTC QLQ-CX24, Questionnaire of Post-traumatic Psychological Disorder, and Greek Symptom Control Questionnaire by M.D. Anderson, in order to assess their QOL. Except for differences in descriptive characteristics of the patients' (age, number of children, contraceptives) and early toxicity in some organs, no statistically significant difference was observed in the main (physical, sexual, emotional) aspects of life between the 2 groups of treated patients. Treatment type had no effect on total QOL. In conclusion, the addition of CHEM to RAD in the treatment plan of CC patients had no significant impact on their QOL.
\end{abstract}

Keywords: quality of life, cervical cancer, treatment, radiotherapy, radiochemotherapy

\section{Introduction}

Gynecological cancers are a frequent group of malignancies in women, accounting for approximately $18 \%$ of all cancers of women worldwide. ${ }^{1}$ Cervical cancer (CC) is the second most deadly gynecological malignancy in the world. In developing countries, 500,000 new cases are diagnosed every year, some of which are the actual cause of death among women of age 35 to 45 years. The CC risk is $1 \%$ during the life of a woman living in a developed country, whereas the corresponding value for a woman living in a country without preventive programs is $5 \% .{ }^{2}$ The development of screening programs and new advances in treatment have contributed greatly to increased survival rates and, in some cases, cure of gynecological cancers. These resulted in a subset of women, known as survivors, who return to their normal functioning and live years after the initial diagnosis without symptoms of the disease. Survivors may experience a wide range of side effects that do not dissipate with time and may persist for a long-term period, such as sexual problems, pain, premature menopause, fatigue, and decreased physical functioning. These side effects can reduce cancer survivors' quality of life (QOL). The recognition that QOL of CC survivors is of great importance led to the emergence of a body of research that has been focusing on QOL issues. Knowledge about QOL issues is crucial to establish follow-up care 
programs adjusted to the survivors' needs and to provide appropriate education in prevention and early detection of survivors' needs, and ultimately improve their QOL. ${ }^{3}$

Patients diagnosed with early stage $\mathrm{CC}$ are usually treated either with surgery (SURG) (complete removal of internal genitals), radiotherapy (RAD) (irradiation of the pelvis and occasionally the para-aortic lymph nodes at target doses of approximately $50 \mathrm{~Gy}$ ), or a combination of both. Some patients also receive concurrent chemotherapy (CHEM) as part of curative treatment. Standard treatment for patients with advanced stages of CC is external pelvic RAD combined with brachytherapy to the cervix and today, usually, combined with CHEM (RAD/CHEM). ${ }^{4-6}$

In our study we decided to compare the QOL of CC patients who were treated with either RAD or RAD/ CHEM. For the estimation of their QOL we used 4 different questionnaires: EORTC QLQ C-30, EORTC QLQ-CX24, Questionnaire of Post-traumatic Psychological Disorder, and Greek Symptom Control Questionnaire by M.D. Anderson. The patients included in this study suffered from CC stages IA-IIIA and filled in the questionnaires 1 year after treatment end. Our aim was to compare the impact of monotherapy (RAD) and combined therapy (RAD/CHEM) on the QOL of CC patients.

\section{Patients and methods}

A total 105 consecutive CC patients (93\% participation) stages IA-IIIA, who met the inclusion criteria, were enrolled to the study; 58 (55\%) had been treated with RAD/CHEM (group 1) and 47 (45\%) with RAD (group 2). Eligible women had squamous cell tumors $<6 \mathrm{~cm}$ at diagnosis and were currently disease-free. Descriptive characteristics of the patients are given in Table 1. All participants were patients in Theagenio Cancer Hospital, Thessaloniki, Greece and filled in the questionnaires 1 year after treatment completion at a scheduled follow-up check. Women who verbally agreed to participate were read an informed consent statement that met criteria defined by the Health Insurance Portability and Accountability Act, as well as by our institution.

In our study the proposed method for patients suffering from squamous cervical cancer, nonresected, stages IA-IIIA (by the International Federation of Gynecology and Obstetrics) is concurrent RAD/CHEM. RAD is applied as external treatment with pelvic fields and $4500 \mathrm{cGy}$, combined with weekly administration of cisplatin, at the radiosensitizer dose of $40 \mathrm{mg} /$ $\mathrm{m}^{2}$ body surface (the therapeutic dose of platinum amounts to $100 \mathrm{mg} / \mathrm{m}^{2}$ body surface) and afterwards brachytherapy, as additional dose. The type of radiation used was photons generated by linear accelerator with $18 \mathrm{MeV}$ energy. In addition, all patients underwent brachytherapy with total dose $3000 \mathrm{cGy}$.

The EORTC QLQ-C30 incorporates 9 multi-item scales: 5 functional scales (physical, role, cognitive, emotional, and social), 3 symptom scales (fatigue, pain, and nausea and vomiting), and a global health and QOL scale. Several singleitem symptom measures are also included. It is internationally considered as a reliable and valid measure of the QOL of cancer patients in multicultural clinical research settings. An essential aspect of the "modular" approach to QOL assessment adopted by the EORTC QLG (Quality of Life Group) is the development of modules specific to tumor site, treatment modality, or a QOL dimension, to be administered in addition to the core questionnaire (EORTC QLQ-C30). The modules, like the core questionnaire,

Table I Descriptive characteristics of patients

\begin{tabular}{|c|c|c|c|c|c|c|c|c|c|c|c|c|c|c|c|}
\hline \multirow[t]{3}{*}{ Variables } & \multicolumn{15}{|c|}{ Group } \\
\hline & \multicolumn{5}{|c|}{ I (RAD/CHEM) } & \multicolumn{5}{|c|}{2 (RAD) } & \multicolumn{5}{|l|}{ Total } \\
\hline & $M / F$ & SD & Min & Max & $\mathbf{N}$ & $\mathbf{M} / \mathbf{F}$ & SD & Min & Max & $\mathbf{N}$ & M/F & SD & Min & Max & $\mathbf{N}$ \\
\hline Age & 49.76 & 9.058 & 33 & 73 & 58 & 61.40 & 12.138 & 35 & 78 & 47 & 54.97 & 2.000 & 33 & 78 & 105 \\
\hline Married & $98 \%$ & & $0 \%$ & $100 \%$ & 58 & $96 \%$ & & $0 \%$ & $100 \%$ & 46 & $97 \%$ & & $0 \%$ & $100 \%$ & 104 \\
\hline $\mathrm{Ch}$ & 1.98 & I.I77 & 0 & 9 & 58 & 2.28 & 1.210 & $\mathrm{I}$ & 5 & 47 & 2.11 & 1.195 & 0 & 10 & 105 \\
\hline Con & $22 \%$ & & $0 \%$ & $100 \%$ & 58 & $18 \%$ & & $0 \%$ & $100 \%$ & 47 & $20 \%$ & & $0 \%$ & $100 \%$ & 105 \\
\hline Con/y & 1.60 & 2.576 & 0 & 10 & 57 & 0.68 & 2.033 & 0 & 10 & 47 & 1.18 & 2.380 & 0 & 10 & 104 \\
\hline $\mathrm{Ab}$ & $30 \%$ & & $0 \%$ & $100 \%$ & 56 & $24 \%$ & & $0 \%$ & $100 \%$ & 40 & $27 \%$ & & $0 \%$ & $100 \%$ & 96 \\
\hline Ab no & 1.02 & 1.053 & 0 & 3 & 47 & 0.28 & 0.815 & 0 & 4 & 36 & 0.70 & 1.021 & 0 & 4 & 83 \\
\hline Pap/y & 4.30 & 2.550 & 0 & 10 & 43 & 4.95 & 3.045 & 0 & 10 & 19 & 4.50 & 2.702 & 0 & 10 & 62 \\
\hline $\mathrm{Pap} / \mathrm{m}$ & 47.38 & 32.446 & I & 120 & 47 & 56.35 & 38.046 & I & 120 & 20 & 50.06 & 34.169 & I & 120 & 67 \\
\hline Stage & $74 \%$ & & IA (0) & IIIA (100) & 58 & $66 \%$ & & IB (0) & IIIA (100) & 47 & $70 \%$ & & $\mathrm{IA}(0)$ & IIIA $(100)$ & 105 \\
\hline Grade & 2.59 & 0.826 & Low & High & 56 & 2.85 & 0.955 & Low & High & 47 & 2.71 & 0.893 & Low & High & 103 \\
\hline
\end{tabular}

Abbreviations: RAD, radiotherapy; RAD/CHEM, radiochemotherapy; M, mean value; F, frequency; SD, standard deviation; Min, minimum value; Max, maximum value; $\mathrm{Ch}$, children; Con, contraceptive use; Con/y, years of contraceptive use; Ab, abortions; Ab no, number of abortions; Pap/y, years before last Pap test; Pap/m, months before last Pap test. 
are designed for use in cancer clinical trials. Specifically for CC, we used the EORTC QLQ-CX24, which consists of 3 multiitem scales and 5 single-item scales. ${ }^{7}$ Two complementary questionnaires were used, the Greek Symptom Control Questionnaire by M.D. Anderson and Questionnaire of Posttraumatic Psychological Disorder, to supplement the assessment of the 2 major questionnaires. Aspects of life examined by the 4 questionnaires are given in Table 2 .

All statistical analysis was performed using SPSS for Windows, version 15.0 (SPSS Inc., Chicago, IL). Groups were compared using nonparametric Mann-Whitney test. We tried to make the design, methodology, statistical analysis, presentation, and interpretation of results in accordance with international standards to ensure reliability and comparability of research results. Reliability test of the questionnaires was based on Cronbach's alpha coefficient. Cronbach's alpha coefficients and ANOVA significance levels are also given in Table 2.

\section{Results}

The results showed that in 2 patients $(2 \%)$ the disease was stage IA, in 19 (18\%) stage IB, in 34 (32\%) IIA, in $29(28 \%)$ IIB, and in 21 (20\%) IIIA. Out of 105 patients, $58(55 \%)$ underwent RAD/CHEM (=group 1) and the other 47 (45\%) only RAD (=group 2). Local recurrences occurred in 33 patients $(31 \%)$ and metastasis in 25 patients $(24 \%)$.
All 4 questionnaires were easily accepted by the participating patients. There were no problems of acceptance, understanding, and response to any question. The results showed that the questionnaires had a high reliability during the sensitivity test (high Cronbach's alpha values, Table 2).

Groups comparisons showed statistically significant differences in descriptive data (RAD patients were older, with more children and fewer years of contraceptive use) and early toxicity in lower digestive system and pelvis, genetic and urinary system, blood and hematological elements: white cells, platelets, hemoglobin, and hematocrit (higher in the RAD/ CHEM group). No statistically significant differences between the 2 groups were observed in general status, physical activity, fatigue, nausea, vomiting, constipation, diarrhea, perception, mood, impact on life and health (EORTC QLQ-30), abdominal spasms, defecation, urination, limb problems, vaginal problems, sexuality, vagina condition during the last week and the last 4 weeks (EORTC QLQ-CX24), post-traumatic stress in the last week due to disease knowledge (Questionnaire of Post-traumatic Psychological Disorder), symptoms' pain intensity, and impact on life in the last 24 hours (Greek Symptom Control Questionnaire by M.D. Anderson).

For the main subject of our study, question 30 of EORTC QLQ-30 (Q30 = Quality of Life), no difference was apparent between groups 1 and 2 (significance level

Table 2 Aspects of life investigated, Cronbach's alpha, and ANOVA P-value for the four questionnaires

\begin{tabular}{|c|c|c|c|c|}
\hline Questionnaire & Questions & Aspect & $\begin{array}{l}\text { Cronbach's } \\
\text { alpha }\end{array}$ & $\begin{array}{l}\text { ANOVA } \\
P \text {-value }\end{array}$ \\
\hline \multirow[t]{9}{*}{ EORTC QLQ C-30 } & $\mathrm{I}-5$ & General status & 0.935 & 0.559 \\
\hline & $6-10$ & Physical activity & 0.934 & 0.321 \\
\hline & $11-13$ & Fatigue & 0.888 & 0.396 \\
\hline & $14-15$ & Nausea, vomiting & 0.973 & 0.553 \\
\hline & $16-17$ & Constipation, diarrhea & 0.701 & 0.445 \\
\hline & $|8-2|$ & Perception & 0.842 & 0.656 \\
\hline & $22-25$ & Mood & 0.895 & 0.693 \\
\hline & $26-28$ & Impact on life & 0.888 & 0.236 \\
\hline & $29-30$ & Health, quality of life & 0.929 & 0.279 \\
\hline \multirow[t]{8}{*}{ EORTC QLQ CX-24 } & Last week & & 0.808 & 0.558 \\
\hline & $31-37$ & Abdominal spasms, defecation, urination & 0.819 & 0.665 \\
\hline & $38-40$ & Limb problems & 0.819 & 0.288 \\
\hline & $41-43$ & Vaginal problems & 0.830 & 0.788 \\
\hline & $44-47$ & Sexuality & & \\
\hline & Last 4 weeks & & 0.829 & 0.558 \\
\hline & $48-49$ & Sexuality & 0.974 & 0.442 \\
\hline & $50-54$ & Vagina condition during last 4 weeks & & \\
\hline Questionnaire of post-traumatic & Metal-22 & Post-traumatic stress in the last week & 0.993 & 0.228 \\
\hline psychological disorder & & due to disease knowledge & & \\
\hline Greek symptom control & MerosIQI-QI5 & Symptoms' pain intensity in the last 24 hours & 0.956 & 0.336 \\
\hline questionnaire by MD Anderson & MerosIlQ16-Q23 & Impact on life in the last 24 hours & 0.933 & 0.668 \\
\hline
\end{tabular}

Notes: Cronbach's alpha coefficient: the closer Cronbach's alpha coefficient is to 10.0 the greater the reliability of the questionnaire; ANOVA P-value: significance level is set at $0.05(P<0.05)$, so any value less than this results in significant effects, while any value greater than this value results in nonsignificant effects. 
$P=0.119>0.005)$, ie, there is no difference in $\mathrm{QOL}$ related to the treatment type followed. This is consistent with the ANOVA results, where the factor "group" was not significant (significance level $P=0.279>0.05$ ). In addition, we observed no significant differences for any of the aspects of life examined (Table 2).

\section{Discussion}

With increasing survival rates of CC patients, healthrelated QOL of the survivors becomes an important issue. QOL is, in this context, defined as a person's self-reported perception of physical, psychosocial, and sexual wellbeing. The different treatment programs affect the lives of surviving women to a varying degree in terms of physical, sexual, and psychosocial functioning. ${ }^{8-14}$ Despite the trend towards more toxic, multimodal treatment regimes, little focus has been put on the long-term effects of therapy. However, it is more common, nowadays, to include QOL measurements in clinical trials.

In our study we compared the QOL of CC patients who were treated with either combined RAD/CHEM or $\mathrm{RAD}, 1$ year after treatment completion. No between-group differences were observed in the total QOL of patients. Statistically significant differences were found in descriptive characteristics (such as age, number of children, and use of contraceptives) and early toxicity in the lower digestive system and pelvis, genetic and urinary system blood, and hematological elements. In the main areas of physical, emotional, and sexual functioning, no significant differences were observed, indicating that the addition of CHEM in the treatment does not significantly affect the QOL of CC patients. It seems that toxicity of treatment is due to RAD.

Our results are in agreement with the conclusion of a study conducted by Greimel et al, which corroborated findings from previous research, in which QOL of survivors treated with adjuvant RAD was compared with QOL of patients treated with SURG with or without adjuvant CHEM. ${ }^{15-17}$ There were no differences between treatment groups in overall health status and QOL, emotional functioning, fatigue, insomnia, and diarrhea. Patients treated with RAD reported more impairment in several domains of QOL, such as physical functioning, role functioning, and cognitive and social functioning. Also, they had more symptoms, such as nausea, vomiting, pain, appetite loss, tight vagina, leaking of urine, and frequent urination than the other 2 groups (SURG and SURG/CHEM). Survivors treated with adjuvant RAD reported more financial difficulties because of their physical condition than women treated with the other 2 treatment modalities. Compared with normative reference data, women treated by SURG with or without CHEM had similar QOL to healthy women, whereas those treated with RAD had lower QOL. The latest group reported a lower sexual activity rate than the other groups of survivors; however, they had similar sexual pleasure and sexual discomfort.

Frumovitz et al compared the QOL and sexual functioning of CC survivors treated with either radical hysterectomy and lymph node dissection or RAD at least 5 years after initial treatment. ${ }^{18}$ Compared with SURG patients and controls using univariate analysis, RAD patients had significantly poorer scores on standardized questionnaires measuring healthrelated QOL (physical and mental health), psychosocial distress, and sexual functioning. The disparity in sexual function remained significant in a multivariate analysis. Their conclusion that RAD is responsible for worse QOL of CC patients agrees with our results.

In a study by Korfage et al, SURG and a combination of therapies (SURG, RAD, and RAD/CHEM) were compared for their effect on the QOL of CC survivors. ${ }^{19}$ After controlling for background characteristics (age, education, job, marital status, children, and country of birth), generic QOL scale scores were similar to those of the reference population, except for worse mental health in survivors. The most frequent symptoms were crampy pain in the abdomen or belly (17\%), urinary leakage (15\%), menopausal symptoms $(18 \%)$, and problems in sexual activity. More sexual worry and worse body image were reported by the 2- to 5 -year survivors than the 6- to 10-year survivors. Compared with SURG only, especially primary RAD was associated with an increased frequency of treatment-related side effects, also after controlling for age and disease stage at diagnosis and follow-up.

According to a recent systematic Cochrane review of acute and late toxicity after concomitant CHEM and RAD, late effects of treatment were not well reported and therefore the effect of CHEM/RAD on these effects could not be determined with confidence. ${ }^{15}$ Currently, the 2 successful strategies in treating CC, concomitant CHEM/RAD and CHEM followed by SURG, are being compared in a large phase III study performed by the European Organization for the Research and Treatment of Cancer (EORTC-55994). One of the objectives in this trial is to compare the QOL of patients under these regimes.

As with all studies based on surveys, response bias could affect results. Selection bias occurs when patients who finally take part in the study have a better QOL than those who are unreachable or refuse to participate. Also, 
since data were collected retrospectively, we do not know whether a QOL difference may have existed in the treatment groups before therapy. Our data suggest that CC survivors treated either with RAD/CHEM or RAD have no significant differences in QOL and physical, psychosocial, and sexual functioning. These findings suggest that CHEM addition in CC treatment does not affect QOL of patients, at least for this particular protocol. Different protocols of treatment or different patients' characteristics could have varying effects on patients' QOL; thus this study should be expanded to other treatment protocols and/or other CC patient groups as well as control groups. By taking into consideration well-established information on long-term QOL outcomes of CC treatments, our data may assist both the patient and the physician in selecting the most appropriate treatment protocol. Since the QOL of CC patients is of great importance, more interest should be focused on the investigation of the effect of treatment type on the survivor's life.

\section{Disclosure}

The authors have no conflicts of interest to disclose in relation to this work.

\section{References}

1. Gonçalves V. Long-term quality of life in gynecological cancer survivors. Curr Opin Obstet Gynecol. 2010;22(1):30-35.

2. Babas E, Ekonomopoulou MT, Karapidaki I, Doxakis A, Betsas G, Iakovidou-Kritsi Z. Indication of participation of caspase-2 and caspase-5 in mechanisms of human cervical malignancy. Int $J$ Gynecol Cancer. 2010;20(8):1381-1385.

3. Stein K, Syrjala K, Andrykowski M. Physical and psychological long-term and late effects of cancer. Cancer. 2008;112(Suppl 11): S2577-S2592.

4. Peters WA 3rd, Liu PY, Barrett RJ 2nd, et al. Concurrent chemotherapy and pelvic radiation therapy compared with pelvic radiation therapy alone as adjuvant therapy after radical surgery in high-risk early-stage cancer of the cervix. J Clin Oncol. 2000;18(8):1606-1613.

5. Ryu HS, Chun M, Chang KH, Chang HJ, Lee JP. Postoperative adjuvant concurrent chemoradiotherapy improves survival rates for high-risk, early stage cervical cancer patients. Gynecol Oncol. 2005;96(2):490-495.
6. Eifel PJ, Winter K, Morris M, et al. Pelvic irradiation with concurrent chemotherapy versus pelvic and para-aortic irradiation for high-risk cervical cancer: an update of radiation therapy oncology group trial (RTOG) 90-01. J Clin Oncol. 2004;22(5):872-880.

7. European Organization for Research and Treatment of Cancer.be. Brussels: EORTC group for research into the Quality of Life. http:// groups.eortc.be/qol/questionnaires_qlqc30.htm. Accessed March 3, 2011.

8. Andersen BL, Anderson B, de Prosse C. Controlled prospective longitudinal study of women with cancer: I. Sexual functioning outcomes. J Consult Clin Psychol. 1989;57(6):683-691.

9. Andersen BL, Anderson B, de Prosse C. Controlled prospective longitudinal study of women with cancer: II. Psychological outcomes. J Consult Clin Psychol. 1989;57(6):692-697.

10. Bergmark K, Avall-Lundqvist E, Dickman PW, Henningsohn L, Steineck G. Vaginal changes and sexuality in women with a history of cervical cancer. N Engl J Med. 1999;340(18):1383-1389.

11. Bruner DW, Lanciano R, Keegan M, Corn B, Martin E, Hanks GE. Vaginal stenosis and sexual function following intracavitary radiation for the treatment of cervical and endometrial carcinoma. Int $J$ Radiat Oncol Biol Phys. 1993;27(4):825-830.

12. Klee M, Thranov I, Machin D. Life after radiotherapy: the psychological and social effects experienced by women treated for advanced stages of cervical cancer. Gynecol Oncol. 2000;76(1):5-13.

13. Jensen PT, Groenvold M, Klee MC, Thranov I, Petersen MA, Machin D. Longitudinal study of sexual function and vaginal changes after radiotherapy for cervical cancer. Int J Radiat Oncol Biol Phys. 2003;56(4):937-949.

14. Klee M, Thranov I, Machin PD. The patients' perspective on physical symptoms after radiotherapy for cervical cancer. Gynecol Oncol. 2000;76(1):14-23.

15. Kirwan JM, Symonds P, Green JA, Tierney J, Collingwood M, Williams CJ. A systematic review of acute and late toxicity of concomitant chemoradiation for cervical cancer. Radiother Oncol. 2003;68(3):217-226.

16. Greimel ER, Winter R, Kapp KS, Haas J. Quality of life and sexual functioning after treatment: a long-term follow-up study. Psychooncology. 2009; 18:476-482.

17. Schover LR, Fife M, Gershenson D. Sexual dysfunction and treatment for early stage cervical cancer. Cancer. 1989;63(1):204-212.

18. Frumovitz M, Sun CC, Schover LR, et al. Quality of life and sexual functioning in cervical cancer survivors. J Clin Oncol. 2005;23(30): 7428-7436.

19. Korfage IJ, Essink-Bot ML, Mols F, van de Poll-Franse L, Kruitwagen R, van Ballegooijen M. Health-related quality of life in cervical cancer survivors: a population-based survey. Int J Radiat Oncol Biol Phys. 2009;73(5):1501-1509.
Cancer Management and Research

\section{Publish your work in this journal}

Cancer Management and Research is an international, peer-reviewed open access journal focusing on cancer research and the optimal use of preventative and integrated treatment interventions to achieve improved outcomes, enhanced survival and quality of life for the cancer patient The journal welcomes original research, clinical \& epidemiological

\section{Dovepress}

studies, reviews \& evaluations, guidelines, expert opinion \& commentary, case reports \& extended reports. The manuscript management system is completely online and includes a very quick and fair peerreview system, which is all easy to use. Visit http://www.dovepress.com/ testimonials.php to read real quotes from published authors. 